\title{
Visualization analysis of research hotspots based on CiteSpace II: taking medical devices as an example
}

\section{Dong-Dong Liu \\ Sheng-Lin Liu \\ Jia-Hua Zhang}

Biomedical Engineering Laboratory, Union Hospital, Tongji Medical College, Huazhong University of Science and Technology, Hubei Province, People's Republic of China
This article was published in the following Dove Press journal:

Medical Devices: Evidence and Research

24 October 2014

Number of times this article has been viewed

\begin{abstract}
Biomedical engineering has been one of the hottest fields in biology and engineering. As an important branch, the medical device has achieved significant progress in the past decades. As a useful method in evaluative bibliometrics, mapping knowledge has been used to explore the trend of one field. In the present study, we retrieve literatures about the medical device from the Web of Science ${ }^{\mathrm{TM}}$ (2004-2013), and acquire 26,793 related records, then analyze time range, region distribution, and main research directions of the literatures, and try to use keywords combined with mapping knowledge to explore the main trends of the medical device, and then aim to provide more information for medical device research. Through the study, we discover: 1) the publications regarding medical devices show an upward trend over the past decade in general; 2) the percentage of publications in the USA $(38.49 \%)$ is the highest all over the world; 3 ) engineering (20.64\%) is the hottest research direction, and takes up about one-fifth of the total publications; 4) the Journal of the American Medical Association and The New England Journal of Medicine are among the two journals that are the most highly cited, followed by Science and The Lancet; and 5) keywords of the medical device include in vitro, quality-of-life, outcomes, management, mortality, depression, and so on. With the help of mapping knowledge, we dig out some hot topics of medical devices and provide more information through trend analysis, and we discover that our findings are related to previous research and further research can enlarge the number of records and optimize the algorithm. We provide a systematic approach for researchers to keep abreast of the development and state
\end{abstract} of the research of medical devices.

Keywords: visualization analysis, medical device, CiteSpace, hotspot

\section{Introduction}

The medical device is an important branch of biomedical engineering, and involves an instrument, apparatus, implant, in vitro reagents, or similar or related article that is used to diagnose, prevent, treat disease, or rehabilitate. ${ }^{1}$ Research about the medical device has achieved significant progress in the past 30 years. Increasing evidence suggests that the medical device plays an important role in clinical diagnostics and makes clinical work more efficient. To meet the growing demands of effective care and accurate diagnosis, a more portable, precise, and sensitive medical device will be designed.

Citation analysis has been used to evaluate the performance of science research and to analyze the hot topic of one field. ${ }^{2}$ Mapping knowledge is a useful method in evaluative bibliometrics, mostly aimed at displaying structural and dynamic aspects of scientific research. ${ }^{3}$ Mapping knowledge has been used to explore the trend of 
fields, such as medical science, ${ }^{4}$ management science, ${ }^{5,6}$ information science, ${ }^{7}$ library science, ${ }^{8}$ and so on.

Chen describes a generic approach to detecting and visualizing emerging trends and transient patterns in scientific literature, and provides a tool named CiteSpace II which enables analysts to perform quantitative and qualitative studies of scientific subject domains more easily. ${ }^{9}$ A lot of literatures about trend analysis using CiteSpace II have been published. However, little research has been done about the hot topics of the medical device by using the bibliometrics methods. In this paper, with the help of CiteSpace II, we analyze literatures about the medical device retrieved from Web of Science, and aim to discover the main trends and hot topics of the medical device over the last decade, then provide more information for medical device research.

\section{Materials and methods}

The data were downloaded from the Web of Science website on February 26, 2014. Under the mode of topic, we used "medical device", "medical equipment", and "medical instrument" separately as subject terms to search the English articles, and chose OR to combine the three data sets. The publication time ranged from 2004 to 2013. In total, 26,793 records were found. Our analysis is a combination of three tools, SPSS, CiteSpace II, and EndNote. We used SPSS 21.0 to generate diagrams and used CiteSpace II to carry out the visualized network, and finally compared the results between CiteSpace II and EndNote.

\section{Results}

\section{Time range}

According to the retrieval results of Web of Science, we obtained the publications about medical devices per year in Web of Science during 2004-2013. As shown in Figure 1,

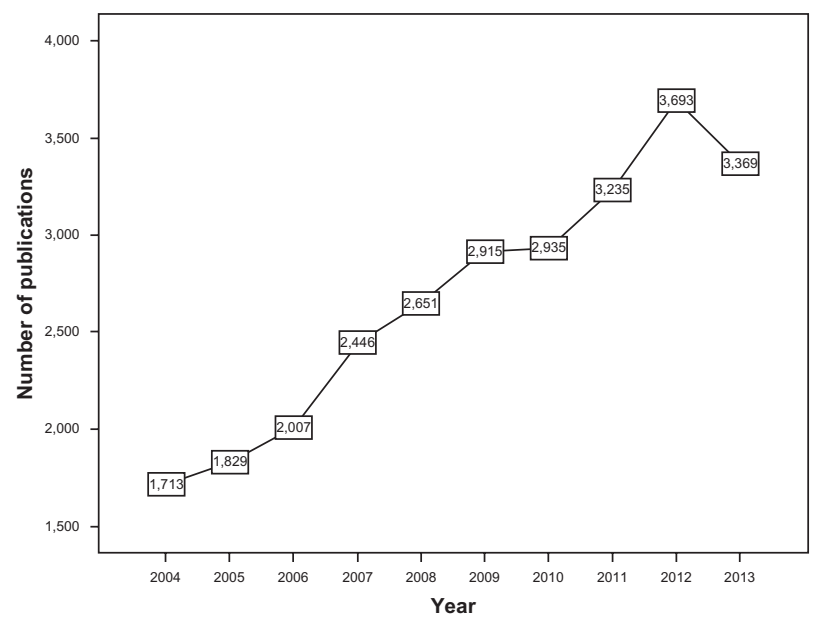

Figure I The number of publications per year, 2004-20I3. the publications of medical device literatures have shown an upward trend over the past decade, from 1,713 papers in 2004 to 3,693 papers in 2012, about twice the former, but the publications in 2013 were on a slight decline. The increase of the number of the literatures indicates that the funding of science and research for medical devices increases, and the medical device attracts more attention around the world.

\section{Region}

Figure 2 illustrates the percentages of publications across countries from 2004 to 2013. The USA (38.49\%) takes up more than a third of the total at the top of the list. The next two significant countries are Germany and England, which are $8.42 \%$ and $6.05 \%$, respectively. People's Republic of China $(3.97 \%)$ only ranks in eighth place, and drops behind Japan $(4.30 \%)$. The large amount of USA literature is closely related to strong economic strength and high research investment; besides, some of the world's leading countries are European. By contrast, People's Republic of China desperately needs to increase the invested funds for scientific research in the field of medical devices and instruments to vitalize the development of the domestic medical device industry.

\section{Research direction}

With the help of the analysis function of Web of Science, we obtained the top ten research directions of the medical device during 2004-2013 (Table 1). Obviously, engineering is still a hot direction, and occupies about one-fifth of the total. Other hot directions include computer science, general internal medicine, surgery, materials science, cardiovascular system cardiology, health care sciences services, physics, radiology nuclear medicine medical imaging, and public environmental occupational health. These findings highlight that the medical device is based on engineering, and involves more and more medical and public health areas.

\section{Citation analyses}

We used CiteSpace II to carry out the visualized analysis on the data. We selected "Article" as the document type from all 26,793 papers on Web of Science, then realigned the search records by times cited - the highest to the lowest, and exported 3,000 records first as our dataset. Various CiteSpace II options were selected. These included 1) the time range: 2004-2013; 2) years per slice: 1 year; and 3) pruning: pathfinder.

Through the co-journal analysis of literatures, we obtained the knowledge mapping network of co-journal literatures in Figure 3. Each node represents a kind of journal. The radius of the node represents the frequency cited. Chen explains 


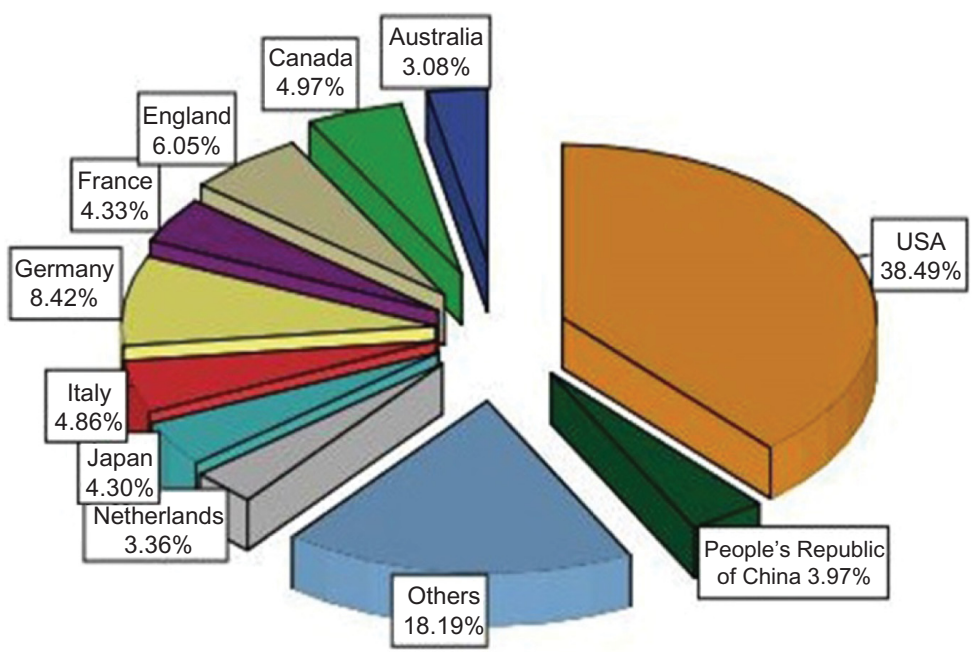

$\square$ Australia
$\square$ Canada
$\square$ England
$\square$ France
$\square$ Germany
$\square$ Italy
$\square$ Japan
$\square$ Netherlands
$\square$ Others
$\square$ People's Republic of China
$\square$ USA

Figure 2 The percentage of publications by country, 2004-2013.

the citation ring in his article "CiteSpace II: Detecting and visualizing emerging trends and transient patterns in scientific literature". ${ }^{9}$ The thickness of a ring is proportional to the number of citations in a given time slice. As shown, the highest cited journal is The New England Journal of Medicine, which has the largest circle, followed by JAMA (The Journal of the American Medical Association) and Science. Other high-cited journals also include The Lancet, Annals of Internal Medicine, Circulation, Nature, and many other kinds of top international magazines.

Figure 4 illustrates a network of co-words of the papers. The highest frequency term is clearly in vitro. Combined with other words, such as quality- of-life, outcomes, survival, management, care, and therapy, we deduce that the research of the medical device emphasizes the concept of patient-oriented medicine.

Table I The top 10 research directions of the medical device, 2004-2013

\begin{tabular}{lll}
\hline Research direction & Number & \% of $\mathbf{2 6 , 7 9 3}$ \\
\hline Engineering & 5,530 & 20.64 \\
Computer science & 1,987 & 7.416 \\
General internal medicine & 1,915 & 7.147 \\
Surgery & 1,869 & 6.976 \\
Materials science & 1,817 & 6.782 \\
Cardiovascular system cardiology & $\mathrm{I}, 806$ & 6.741 \\
Health care sciences services & $\mathrm{I}, 688$ & 6.3 \\
Physics & $\mathrm{I}, 40 \mathrm{I}$ & 5.229 \\
Radiology nuclear medicine & $\mathrm{I}, 310$ & 4.889 \\
medical imaging & & \\
Public environmental & $\mathrm{I}, 19 \mathrm{I}$ & 4.445 \\
occupational health & & \\
\hline
\end{tabular}

Patient-centered medicine and patient-oriented research improve health outcomes for individual patients. ${ }^{10}$ In the meantime, types of words such as reliability, validity, and risk-factors remind us that the medical device is more and more concerned with reliability during clinical practice.

From Table 1, we clearly observe that publications about engineering occupy one-fifth of the total. In order to further discover keywords of engineering, we exported the top 2,000 records from all 5,530 records sorted by times cited - the highest to the lowest. Figure 5 shows a network of co-words of the engineering field. Compared

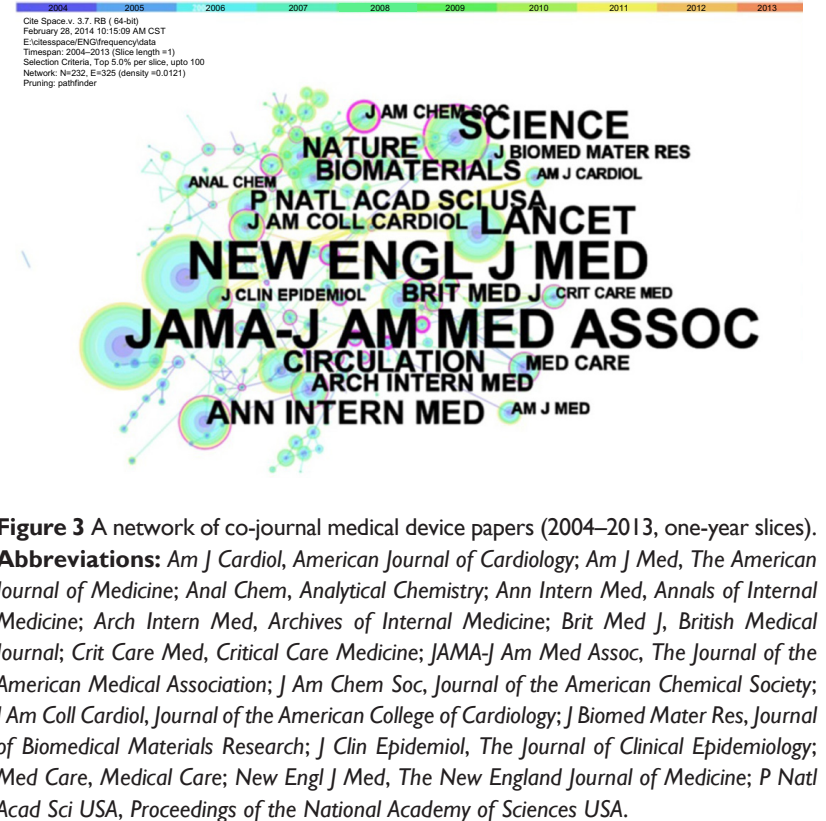




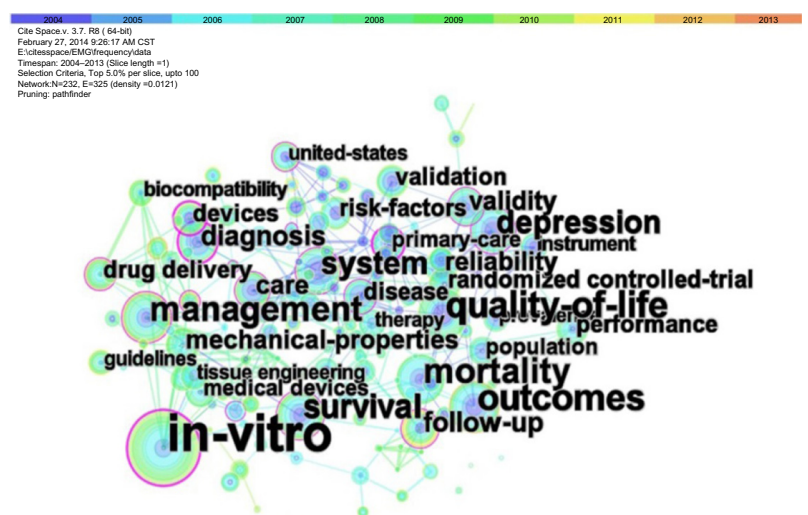

Figure 4 A network of co-words of medical device papers (2004-20I3, one-year slices).

with Figure 4, some new words appear, such as adhesion, design, polymer, and films. Meanwhile, some words are not obvious in Figure 4, and their appearance frequency becomes high, such as biocompatibility and mechanicalproperties.

\section{Discussion}

In this paper, we present the publication time range, country, research direction, and citation analysis of the papers published in the field of the medical device from 2004 to 2013. From research direction aspects, we observe that engineering is still the hottest direction of the medical device. However, more and more clinical medicine and public health researches infiltrate the research of the medical device, which reflects the fact that more attention is being paid to medical device design and research that will yield practical results in the clinic. The development of practical, effective, and safe devices depends on a perfect understanding and a close follow-up of medical devices in clinical application to ensure medical devices can run properly and safely.

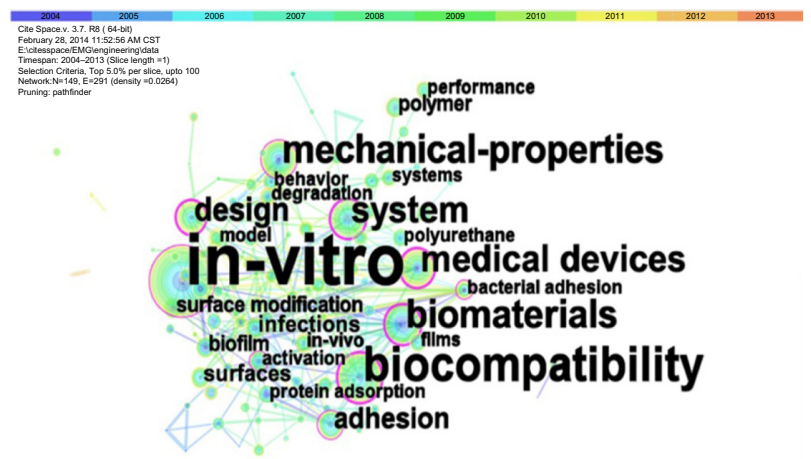

Figure 5 A network of engineering field co-words from medical device papers (2004-2013, one-year slices).
Meanwhile, we used analysis tools in EndNote for analyzing the same dataset mentioned in the co-word analysis. In EndNote, we selected fields including "title", "short title", "keywords", and "abstract" to obtain subject terms. The top 20 terms and their records are listed in Table 2. From Table 2 and Figure 4, we can easily notice that most of the subject terms in Table 2 appear in Figure 4, and the records of subject terms are proportionate to the size of corresponding node. Therefore, we confirm that EndNote and CiteSpace II are in good agreement with the subject terms results.

Herman and Devey forecasts the trend in medical device technologies, and identifies six major technology themes that consist of: 1) electronics technology; 2) detection, diagnosis, and monitoring technologies; 3) decentralized care technologies; 4) minimally invasive technologies; 5) synthetic organs, tissues, and combination device/biological and device/drug technologies; and 6) demographically oriented technologies. ${ }^{11}$ In our study, combining Figures 4 and 5, we discover our finding is in accordance with Herman and Devey's, such as "in vitro" - diagnosis technologies; "surgery" - minimally invasive technologies; "biocompatibility, mechanical-properties" - synthetic organs; "tissue engineering, surface modification" - tissues; "drug deliver" - drug technologies. However, our research has not completely covered the six themes, possibly connected with the dataset we selected. We analyzed just 3,000 records from all 26,793; further research can enlarge the number of records and optimize the algorithm. It is undeniable that there are a few shortcomings in this paper. Medical

Table 2 The top 20 subject terms from EndNote

\begin{tabular}{ll}
\hline Terms & Records \\
\hline Quality-of-life (including quality of life) & 239 \\
Care & 115 \\
Outcomes & 115 \\
Management & 112 \\
In vitro & 111 \\
Biomaterials & 107 \\
System & 103 \\
Reliability & 103 \\
Surgery & 101 \\
Validity & 92 \\
Mortality & 89 \\
Depression & 88 \\
Therapy & 83 \\
Performance & 85 \\
Validation & 83 \\
Prevalence & 81 \\
Disease & 77 \\
Devices & 76 \\
Complications & 71 \\
Questionnaire & 69 \\
\hline
\end{tabular}


device research is a relatively broad field, and though we selected multiple similar subject terms and tried to use the topic mode to expand the retrieval, in fact there was still a fraction of publications that might not be in our range. When we chose to reach a balance between the precision and recall of retrieval, we had to sacrifice a small part of the publications. It would be beneficial to do further work to optimize retrieval strategy. Besides, we only used Web of Science, not other databases, which possibility limited the diversity of results. To sum up, this paper demonstrates the general trends and research hotspots about the field of the medical device to some extent. Our findings provide an approach that visualization analysis is available to explore research hotspots in one field.

Thanks to mobile Internet, wearable devices, big data, and a series of new generation technology, medical devices have been completely reversed. These new technologies are fully subverting our understanding of previous medical treatment. Predictably, these new technologies will advance the research and design of medical devices and change current clinical practice patterns.

\section{Conclusion}

There is no doubt that medical devices have made huge developments in the past decade. Owing to the progress from information technology and molecular biology, medical devices will enter into a new stage.

Obviously, with the help of mapping knowledge, we can dig out some hot topics of a field and provide much information during trend analysis. We believe our work presents an in-depth analysis of major studies of medical devices and a systematic method that researchers can use as frequently as needed to keep abreast of the development of the research of medical devices.

\section{Acknowledgments}

This work was supported by a grant from the National Natural Science Foundation of China (No 81371102).

\section{Disclosure}

The authors report no conflicts of interest in this work.

\section{References}

1. Nahler G. Medical device. Dictionary of Pharmaceutical Medicine. 2009:111.

2. Baker DR. Citation analysis: A methodological review. Soc Work Res Abstr. 1990;26(3):3-10.

3. Noyons ECM, Moed HF, Luwel M. Combining mapping and citation analysis for evaluative bibliometric purposes: A bibliometric study. JAm Soc Inform Sci. 1999;50(2):115-131.

4. Chen $\mathrm{C}, \mathrm{Hu} \mathrm{Z}$, Liu S, Tseng $\mathrm{H}$. Emerging trends in regenerative medicine: a scientometric analysis in CiteSpace. Expert Opin Biol Ther. 2012;12(5):593-608.

5. Hou J, Chen Y. Research on visualization of the evolution of strategic management front. Stud Sci Sci. 2007;6:16-17.

6. Gundolf K, Filser M. Management research and religion: a citation analysis. J Bus Ethics. 2013;112(1):177-185.

7. White HD, McCain KW. Visualizing a discipline: an author co-citation analysis of information science, 1972-1995. J Am Soc Inform Sci. 1998;49(4):327-355.

8. Rongying Z, Ju W. Knowledge Mapping Analysis of Library Science [J]. J Libr Sci China. 2011;2:005.

9. Chen C. CiteSpace II: Detecting and visualizing emerging trends and transient patterns in scientific literature. J Am Soc Inform Sci Tech 2006;57(3):359-377.

10. Sacristán JA. Patient-centered medicine and patient-oriented research: improving health outcomes for individual patients. BMC Med Inform Decis Mak. 2013;13:6.

11. Herman WA, Devey GB. Future Trends in Medical Device Technologies: a Ten-Year Forecast. Food and Drug Administration, Center for Devices and Radiological Health; 2011. Available from: http://www.fda gov/downloads/AboutFDA/.../CDRH/.../UCM238527.pdf. Accessed September 8, 2014.
Medical Devices: Evidence and Research

\section{Publish your work in this journal}

Medical Devices: Evidence and Research is an international, peerreviewed, open access journal that focuses on the evidence, technology, research, and expert opinion supporting the use and application of medical devices in the diagnosis, treatment and management of clinical conditions and physiological processes. The identification of novel

\section{Dovepress}

devices and optimal use of existing devices which will lead to improved clinical outcomes and more effective patient management and safety is a key feature. The manuscript management system is completely online and includes a quick and fair peer-review system. Visit http://www. dovepress.com/testimonials.php to read real quotes from authors. 